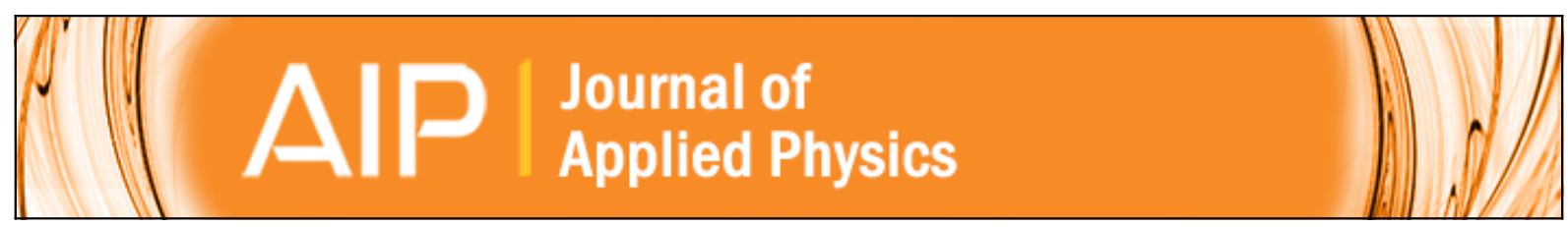

\title{
Electron mobility in CdO films
}

S. K. Vasheghani Farahani, T. D. Veal, P. D. C. King, J. Zúñiga-Pérez, V. Muñoz-Sanjosé, and C. F. McConville

Citation: Journal of Applied Physics 109, 073712 (2011); doi: 10.1063/1.3562141

View online: http://dx.doi.org/10.1063/1.3562141

View Table of Contents: http://scitation.aip.org/content/aip/journal/jap/109/7?ver=pdfcov

Published by the AIP Publishing

$\underset{\substack{\text { Publoning } \\ \text { pulp }}}{A}$ Re-register for Table of Content Alerts

Create a profile.

Sign up today! 


\title{
Electron mobility in CdO films
}

\author{
S. K. Vasheghani Farahani, ${ }^{1}$ T. D. Veal, ${ }^{1, a)}$ P. D. C. King, ${ }^{2}$ J. Zúñiga-Pérez, ${ }^{3}$ \\ V. Muñoz-Sanjosé, ${ }^{4}$ and C. F. McConville $\left.{ }^{1, b}\right)$ \\ ${ }^{1}$ Department of Physics, University of Warwick, Coventry CV4 7AL, U. K \\ ${ }^{2}$ School of Physics and Astronomy, University of St Andrews, St Andrews KY16 9SS, U. K. \\ ${ }^{3}$ Centre de Recherche sur l'Hétéro-Epitaxie et ses Applications, Centre National de la Recherche Scientifique, \\ Parc de Sophia Antipolis, Rue Bernard Grégory, 06560 Valbonne, France \\ ${ }^{4}$ Departamento de Fisica Aplicada y Electromagnetismo, Universitat de Valéncia, C/Dr. Moliner 50, 46100 \\ Burjassot, Spain
}

(Received 31 January 2011; accepted 9 February 2011; published online 6 April 2011)

\begin{abstract}
Electron mobility in degenerate $\mathrm{CdO}$ thin films has been studied as a function of carrier concentration. The "optical" mobility has been determined from infrared reflectance measurements of the conduction band plasmon lifetime. The acquired values vary from $\sim 209$ to $\sim 1116 \mathrm{~cm}^{2} \mathrm{~V}^{-1} \mathrm{~s}^{-1}$ for carrier concentrations between $2.5 \times 10^{20}$ and $2.6 \times 10^{19} \mathrm{~cm}^{-3}$. Ionized impurity scattering is shown to be the dominant effect reducing the intra-grain mobility of the electrons at room temperature. The transport mobilities from Hall effect measurements range between $\sim 20$ and $\sim 124 \mathrm{~cm}^{2} \mathrm{~V}^{-1} \mathrm{~s}^{-1}$ which are much lower than the optical mobilities. Simulation of grain boundary scattering-limited mobility is commonly based on models that assume a depletion layer at the boundaries which causes an inter-grain potential barrier. These models are found not to be applicable to $\mathrm{CdO}$ as it has been previously shown to have surface electron accumulation. Therefore, simulation of the transport mobility has been performed using the Fuchs-Sondheimer and Mayadas-Shatzkes models to take into account the grain boundary and surface scattering mechanisms, in addition to intra-grain scattering. The results indicate that electron scattering at grain boundaries with $\sim 95 \%$ reflection is the dominant mechanism in reducing the mobility across the layer. The effect of surface scattering plays only a minor role in electron transport. (c) 2011 American Institute of Physics. [doi:10.1063/1.3562141]
\end{abstract}

\section{INTRODUCTION}

Carrier transport in semiconductors is of great importance, from studying fundamental physical phenomena to their application in optical and electronic devices. The operation of a transparent conducting oxide (TCO) is based on the band structure-related physical quantities, such as bandgap and bandedge effective mass, as well as the carrier collisions within the material represented by the corresponding carrier lifetimes for elastic scattering. CdO is a group II-VI transparent conductor possessing a relatively low bandgap and high bulk conductivity relative to other TCOs. Koffyberg ${ }^{1}$ studied the Hall mobility of $\mathrm{CdO}$ and suggested that scattering by ionized donors may be the dominant process in limiting the mobility. Indeed, it can be deduced from the recent work by Speaks et $a l^{2}$ that, for carrier concentrations well below $5 \times 10^{20} \mathrm{~cm}^{-3}$ which corresponds to the Fermi level stabilization of $\mathrm{CdO}$, donor defects are dominant.

Yan et $a l^{3}{ }^{3}$ investigated the mobility of $\mathrm{CdO}$ samples with different Sn-doping levels in which the effect of ionized impurity and grain boundary scattering was suggested to play the major role in electron transport. On the other hand, several authors ${ }^{4-6}$ have studied the transport mobility by taking into account the grain boundary scattering for nondegenerate and degenerate ${ }^{7}$ semiconductors based on Seto's model $^{8}$ wherein a depletion layer exists at the boundaries. $\mathrm{CdO}$, having donor-type surface states and an accumulation

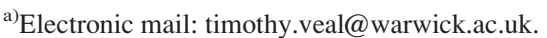

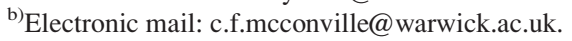

layer $^{9}$ at the grain boundaries, cannot be analyzed by the aforementioned methods. Therefore, we have applied the Fuchs-Sondheimer (FS) ${ }^{10,11}$ and Mayadas-Shatzkes (MS) ${ }^{12}$ models to understand the nature of scattering mechanisms in our degenerate $\mathrm{CdO}$ samples.

\section{EXPERIMENTAL DETAILS}

In this work, cadmium oxide layers with (001) orientation were grown on $r$-plane sapphire at atmospheric pressure by metal-organic vapor-phase epitaxy in which the precursors for oxygen and cadmium were tertiary butanol and dimethylcadmium respectively. The optoelectronic properties of degenerate $\mathrm{CdO}$ have been studied for as-grown samples and after reducing the carrier concentration, $n_{b}$, by annealing in vacuum at $400{ }^{\circ} \mathrm{C}$ for 2 to $24 \mathrm{hrs}$.

Optical measurements were performed using a Bruker Vertex 70v Fourier-transform infrared spectrometer over the energy range 0.1 to $2.7 \mathrm{eV}$. Both the transmittance and reflectance of the samples were measured using an incident angle of 11 degrees with respect to the surface normal while maintaining the same optical path length. This allowed the conversion of transmission in the visible region to absorption spectra without requiring assumptions concerning the energy dependence of the optical reflectance, as is commonly the case. Hall effect, based on the standard Van der Pauw configuration, was used to obtain the sheet density, $n_{s}$, and transport mobility of the $\mathrm{CdO}$ samples. Both the optical and Hall effect measurements were carried out at room temperature. 
X-ray diffraction (XRD) and atomic force microscopy (AFM) measurements have been performed, before and after annealing, for structural characterization. The full width at half maximum of the $\mathrm{CdO} 002$ rocking curve was in the range of $0.27-0.29^{\circ}$ for the investigated samples, indicating a threading dislocation density of the order of $2-4 \times 10^{9}$ $\mathrm{cm}^{-2} \cdot{ }^{13,14}$ This also implies that the density of dislocations in the samples is independent of the annealing treatment performed after growth. In the AFM images, pyramidal grains several hundreds of nanometers in size were observed which are similar to those previously reported for samples grown by the same method. ${ }^{15}$

\section{DATA, SIMULATION, AND ANALYSIS}

\section{A. Bandgap and bandedge effective mass}

Infrared (IR) reflectance of epitaxial $\mathrm{CdO}$ on $r$-plane sapphire within an energy range of 100 to $900 \mathrm{meV}$ for three different carrier concentrations is shown in Fig. 1. The energy corresponding to minimum reflectance, which is related to the conduction band plasma resonant energy, increases with carrier concentration. In order to model the spectra, a three layer stratified medium (vacuum/CdO/sapphire) with coherent interference ${ }^{16}$ has been considered. The two oscillator model of the complex dielectric function is used for the $\mathrm{CdO}$ layer, while the factorized model was applied for the sapphire substrate. ${ }^{17}$ The high frequency dielectric constant, $\varepsilon(\infty)$, for $\mathrm{CdO}$ was set to $5.3^{18}$ which is consistent with a recent investigation of optical constants of $\mathrm{CdO}$ over a range of energies. ${ }^{19}$ The high frequency dielectric constant of 3.06 was used for sapphire. ${ }^{20}$ Simulation of the data, as shown in Fig. 1, allowed the determination of the plasma resonant energy and broadening, and the film thickness, $d$. The carrier concentration of the

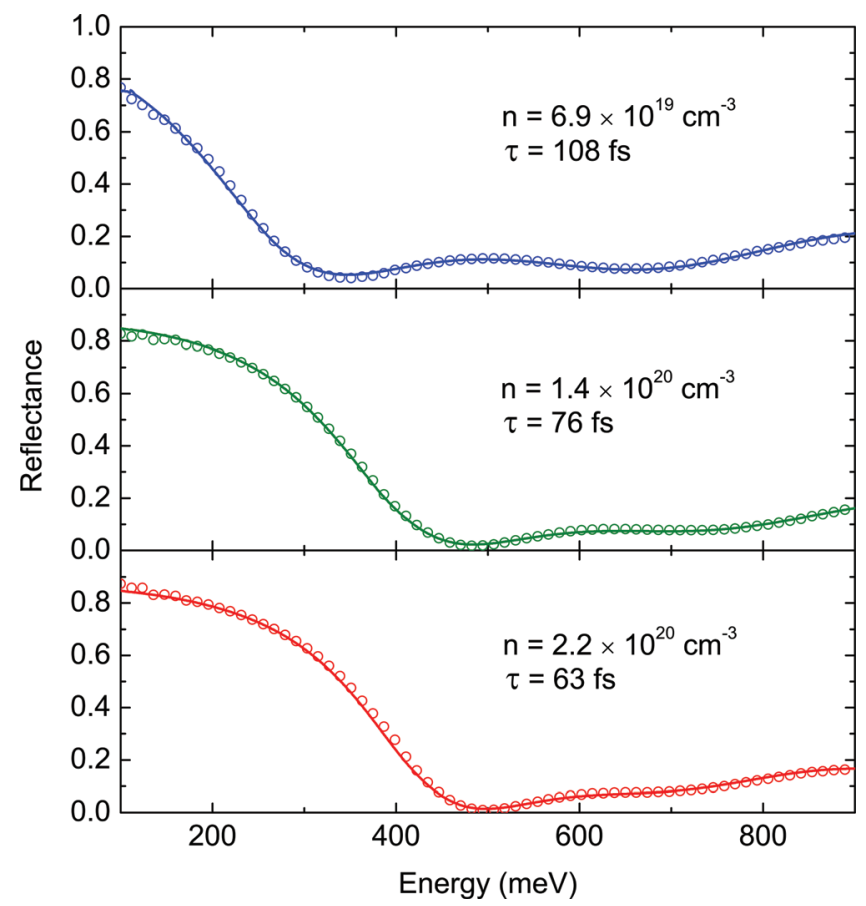

FIG. 1. (Color online) Mid-IR reflectance spectra (open circles) and respective simulations (solid lines) of three $\mathrm{CdO}$ samples with different carrier concentrations and lifetimes. samples was calculated via, $n=n_{s} / d$, which varies between $2.6 \times 10^{19}$ and $2.5 \times 10^{20} \mathrm{~cm}^{-3}$.

By analyzing the evolution of plasma energy and optical absorption edge with carrier density by performing model carrier statistics calculations employing a k.p nonparabolic dispersion ${ }^{21}$ and bandgap renormalization effects, the fundamental bandgap and bandedge effective mass were found to be $2.20 \mathrm{eV}$ and $0.24 m_{0}$, respectively. These are similar to the results of an earlier investigation by Jefferson et al. which included some of the same samples investigated here. ${ }^{22}$ The small differences are attributed to the larger range of bulk carrier densities and more accurate determination of the absorption coefficient utilized here.

\section{B. Optical mobility}

Broadening of the plasma edge obtained from mid-IR reflectance simulations has been used to determine the optical carrier lifetime, $\tau$. The optical mobility of each sample has been calculated using optical carrier lifetime and the density of states averaged effective mass of conduction electrons, $\left\langle m^{*}\right\rangle$, according to the equation, $\mu_{\text {opt }}=e \tau /\left\langle m^{*}\right\rangle$. The results versus carrier concentration are plotted in Fig. 2 which show that the optical mobility varies between $\sim 209 \mathrm{~cm}^{2} \mathrm{~V}^{-1} \mathrm{~s}^{-1}$ for the highest and $\sim 1116 \mathrm{~cm}^{2} \mathrm{~V}^{-1} \mathrm{~s}^{-1}$ for the lowest carrier concentrations. With increasing carrier concentration, the broadening of the plasma edge increases as seen in Fig. 1 which corresponds to the reduction of the optical carrier lifetime. This phenomenon causes the optical mobility to decrease with carrier concentration as shown in Fig. 2.

The degenerate form of the Brooks-Herring formula ${ }^{23}$ has been used to simulate the ionized impurity scatteringlimited mobility of the samples as a function of carrier concentration:

$$
\mu_{\text {ion }, \mathrm{deg}}=\frac{24 \pi^{3}\left(\varepsilon_{s} \varepsilon_{0}\right)^{2} \hbar^{3} n}{e^{3} m^{* 2} N_{\text {ion }}\left[\ln [1+y(n)]-\frac{y(n)}{1+y(n)}\right]},
$$

where,

$$
y(n)=\frac{2\left(3 n \pi^{8}\right)^{1 / 3} \hbar^{2} \varepsilon_{s} \varepsilon_{0}}{e^{2} m^{*}},
$$

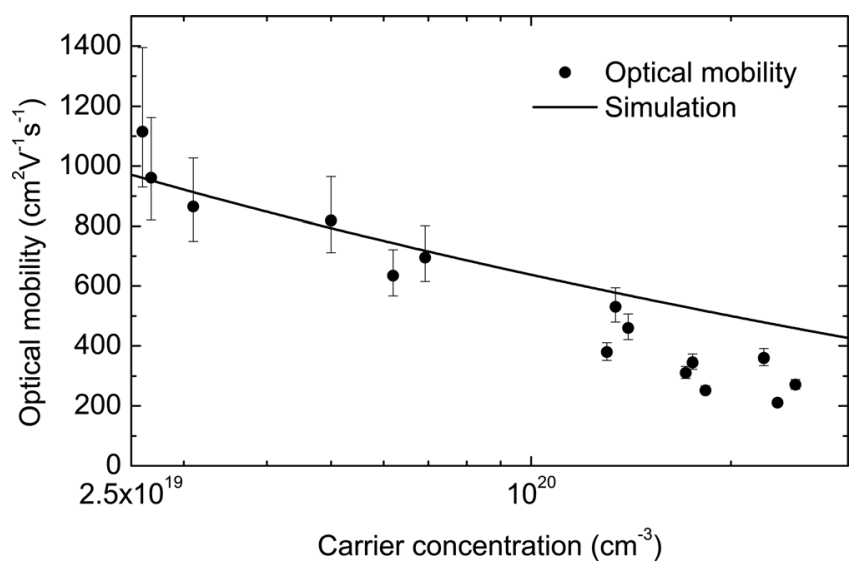

FIG. 2. Optical mobility of $\mathrm{CdO}$ and simulation of the ionized impurity scattering-limited mobility. 
where $m^{*}$ is the density of states averaged effective mass, and $N_{i o n}$ is the density of ionized impurities. The highest Fermi energy of the samples investigated is $\sim 0.48 \mathrm{eV}$ above the conduction band minimum (CBM) while the Fermi level stabilization energy or charge neutrality level of $\mathrm{CdO}$ lies $1 \mathrm{eV}$ above CBM. ${ }^{2}$ Therefore, $N_{\text {ion }}$ in Eq. (1) has been approximated by the density of ionized donors. The ionized donors have been assumed to be singly charged. The simulation of the optical mobility data points is represented in Fig. 2. The results show that ionized impurity scattering is the dominant scattering mechanism reducing the optical mobility in degenerate $\mathrm{CdO}$ at room temperature. The effect of lattice scattering on the mobility was found to be negligible.

\section{Transport mobility}

The transport mobility of degenerate $\mathrm{CdO}$ samples measured by the Hall effect varies between $\sim 20$ and $\sim 124$ $\mathrm{cm}^{2} \mathrm{~V}^{-1} \mathrm{~s}^{-1}$ for the highest and lowest carrier concentrations, respectively, as shown in Fig. 3. It is apparent that the transport mobility is significantly lower than the optical mobility of the electrons for all the samples. Therefore, during Hall effect measurements of the transport mobility, the conduction electrons must experience one or more additional scattering mechanisms. While during transport measurements the electrons traverse the film in response to the applied bias, optical excitation during IR reflectance results in no such phenomenon. This suggests that grain boundary scattering and/or surface scattering are responsible for the transport mobility being much lower than the optical mobility. This interpretation is justified by the following quantitative modeling of these electron scattering mechanisms.

Commonly, the effect of grain boundaries on carrier transport mobility has been studied based on inter-grain barriers resulting from boundary trap states which are indicative of depletion layers at the boundaries. Simulation of the transport mobility under such conditions is based on the model proposed by $\operatorname{Seto}^{8}$ for nondegenerate semiconductors. Under the same boundary conditions with degenerate intra-grain

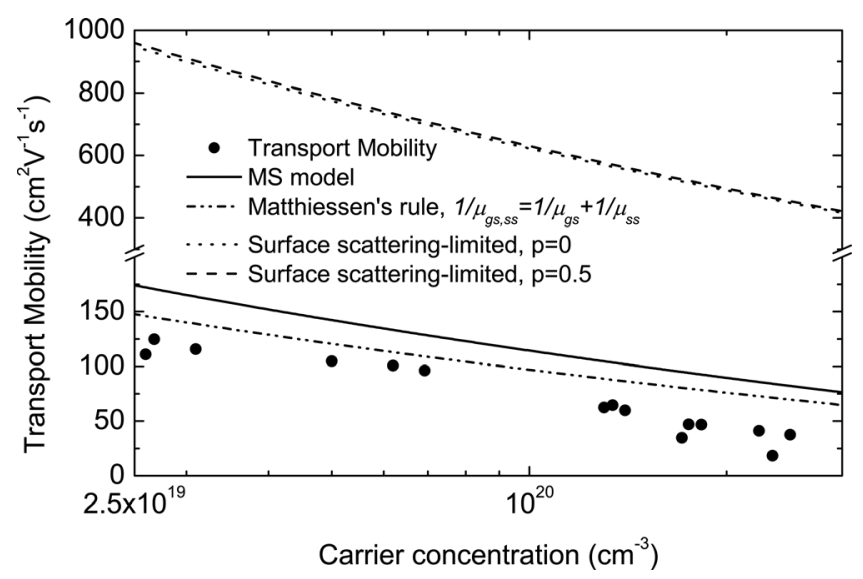

FIG. 3. Transport mobility of $\mathrm{CdO}$ and simulation via the MS model along with the application of Matthiessen's rule to grain boundary and surface scattering-limited mobilities; the effect of surface scattering alone using FS theory is represented which shows the minor influence of surface scattering on the transport mobility. The error bars of the data lie within the solid points. characteristics, the modified expression for grain boundary scattering-limited mobility incorporating Fermi-Dirac statistics is used which predicts that the transport and optical mobility will merge as the barrier becomes surmountable by increasing the carrier concentration and therefore the Fermi level. However, as can be seen in Figs. 2 and 3, the two sets of data points do not merge at higher carrier concentrations. This suggests that this approach to grain boundary scattering is not appropriate here.

Indeed, previous investigations have shown that $\mathrm{CdO}$ has a surface accumulation layer. ${ }^{9}$ Therefore, we expect that there exists an electron accumulation at the grain boundaries and dislocation lines within the films. This has been implemented in the modeling of the grain boundary scattering. In order to simulate the transport mobility of the $\mathrm{CdO}$ samples, we therefore additionally considered scattering by grain boundaries and surfaces making use of the MS model ${ }^{12}$ together with the FS theory of scattering at surfaces which apply to a degenerate electron gas. ${ }^{10,11}$ It is worthy of mention that from the analysis of the optical absorption and midIR reflectance data, it is apparent that there exists a BursteinMoss shift for all the $\mathrm{CdO}$ samples investigated, indicating the relevance of considering the conduction electrons as a degenerate electron gas. This feature and the aforementioned electron accumulation make these models applicable to simulate the data.

Firstly, the effect of surface scattering has been modeled according to the equation: ${ }^{11}$

$$
\mu_{s s}=\mu_{0}\left[1-\frac{3}{2 k}(1-p) \int_{1}^{\infty}\left(\frac{1}{t^{3}}-\frac{1}{t^{5}}\right) \frac{1-e^{-k t}}{1-p e^{-k t}} d t\right],
$$

where, $\mu_{0}$ is the bulk mobility, $k$ is the ratio of film thickness, $d$, to the mean free path of the electrons in the intragrain domains, $l$, and $p$ is the fraction of the electrons being scattered specularly from the surfaces. The bulk mobility has been taken to be the ionized impurity scattering-limited mobility as deduced from the optical mobility studies. The average mean free path in the bulk has been calculated to be $\sim 35 \mathrm{~nm}$ using the average bulk relaxation time obtained from mid-IR reflectance simulation, and the calculated density-of-states-averaged electron velocity. The average film thickness has been determined to be $\sim 480 \mathrm{~nm}$. The respective model curves, in which the limiting effect of diffuse collisions at the surfaces, $p=0$, is compared to that of $50 \%$ specular reflection, are shown in Fig. 3. The result implies that collisions at the surfaces, either diffuse or specular, would not result in a considerable change to the optical mobility. This is because the average mean free path within the grains is much smaller than the thickness of the films.

In the MS model, a Gaussian distribution of planar grain boundaries as $\delta$-function potentials is used with an average spacing. Boltzmann's equation is solved for this configuration including a relaxation time, $\tau$, corresponding to the bulk scattering mechanism. Surface scattering is included by applying the surface boundary conditions on the electron distribution function. ${ }^{10}$ Thus, the following relation has been used to model the transport mobility: ${ }^{12}$ 


$$
\begin{aligned}
\mu_{t r}= & \mu_{g s}-\frac{6 \mu_{0}}{\pi k}(1-p) \int_{0}^{\pi / 2} d \varphi \int_{1}^{\infty} \frac{\cos ^{2} \varphi}{G^{2}(t, \varphi)} \\
& \times\left(\frac{1}{t^{3}}-\frac{1}{t^{5}}\right) \frac{1-e^{-k t G(t, \varphi)}}{1-p e^{-k t G(t, \varphi)}} d t,
\end{aligned}
$$

in which,

$$
\begin{aligned}
\mu_{g s} & =\mu_{0}\left[1-1.5 \alpha+3 \alpha^{2}-3 \alpha^{3} \ln \left(1+\alpha^{-1}\right)\right], \\
G(t, \varphi) & =1+\alpha /\left[\cos \varphi\left(1-t^{-2}\right)^{1 / 2}\right], \\
\alpha & =(l / g)[R /(1-R)],
\end{aligned}
$$

where $\mu_{g s}$ is the grain boundary scattering-limited mobility, $g$ is the average lateral grain size, $R$ is the fraction of the electrons being reflected from grain boundaries.

From XRD data, a threading dislocation density of the order of $2-4 \times 10^{9} \mathrm{~cm}^{-2}$ was obtained. If a homogeneous and isotropic dislocation distribution is assumed, this density results in a dislocation every $200 \mathrm{~nm}$ along the two perpendicular in-plane $\langle 100\rangle$ directions. Furthermore, this typical length is consistent with the AFM images, in which pyramidal grains several hundreds of nanometers in size are observed. Therefore, a length of $200 \mathrm{~nm}$ has been used as the average separation of the $\delta$-function potential barriers considered as the lateral grain dimension within the MS model. Simulation of the transport mobility using the combined three scattering mechanisms in the MS model, Eq. (3), is shown in Fig. 3. A high reflection coefficient of $R \sim 0.95$ at the boundaries is required to obtain a model curve consistent with the measured mobilities. The negligible contribution of surface scattering to the transport mobility is apparent in the figure.

Also, the result of applying Matthiessen's rule to separately calculated surface and grain boundary scattering-limited mobilities, via Eqs. (2) and (4), is represented in Fig. 3. The respective model curve shows a slight deviation from that of the MS model which is caused by the fact that the bulk scattering mechanism is incorporated within the calculations of the FS and MS models. In any case, it is apparent from the modeling results that grain boundary scattering is the dominant mechanism in limiting the transport mobility.

The variation of the transport mobility with grain size, predicted from the modeling, is represented in Fig. 4 for three different carrier concentrations. It is apparent that, as the grain size increases, the transport mobility tends to the bulk mobility within the grain. The mobility rises more steeply with increasing grain size when the grain size is low and closer to the bulk mean free path. So a large improvement of transport mobility is expected for a slight increase from the current grain size, with the rate of improvement diminishing as the grain size becomes much greater than the mean free path. This indicates that small improvements in the growth will have a relatively large effect on the mobility of $\mathrm{CdO}$ films.

Finally, it should be noted that for higher carrier concentrations, the measured mobilities deviate somewhat from the model curves, as can be seen in Figs. 2 and 3 for optical and transport mobilities, respectively. As the formation energy of acceptors decreases and that of donors increases as the Fermi level increases toward the charge neutrality level, ${ }^{2}$ the den-

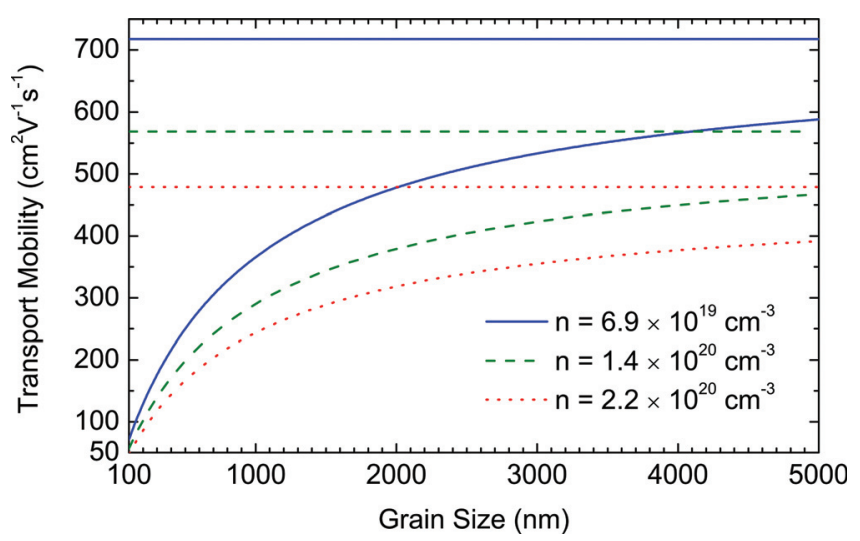

FIG. 4. (Color online) Modeling of the transport mobility as a function of grain size for three $\mathrm{CdO}$ samples with different carrier concentrations and reflection coefficient, $R=0.95$. Intra-grain mobilities for respective carrier concentrations are represented as horizontal lines.

sity of acceptors may no longer be negligible. Therefore, at high carrier densities, in addition to the ionized donors reducing the mobility, there may also be ionized acceptors acting as scattering centers. This partial compensation behavior, as previously observed for $\mathrm{InN},{ }^{24}$ would account for the deviation at high carrier density from the model curves in which only donors have been taken into account.

\section{CONCLUSIONS}

The optical mobility of the $\mathrm{CdO}$ samples ranges from $\sim 209$ to $\sim 1116 \mathrm{~cm}^{2} \mathrm{~V}^{-1} \mathrm{~s}^{-1}$ for carrier concentrations ranging between $2.5 \times 10^{20}$ and $2.6 \times 10^{19} \mathrm{~cm}^{-3}$. Ionized impurity scattering has been observed to be the dominant mechanism limiting the intra-grain mobility of $\mathrm{CdO}$ layers at room temperature. The transport mobilities ranging between $\sim 20$ and $\sim 124 \mathrm{~cm}^{2} \mathrm{~V}^{-1} \mathrm{~s}^{-1}$ are significantly lower than the optical mobilities. As the bulk mean free path of the electrons is small compared to the film thickness, surface scattering is not a significant mobility reducing mechanism. Instead, scattering at grain boundaries is the prevailing mechanism reducing the electron transport mobility. The commonly used models of grain boundary scattering for simulation of the transport mobility are not applicable to $\mathrm{CdO}$ as they assume a depletion layer at the grain boundaries. Given that $\mathrm{CdO}$ has a surface accumulation layer, which implies that electron accumulation is expected to take place at dislocations and grain boundaries, the effect of grain boundary scattering is simulated using alternative models. Simulation of the transport mobility shows that transport scattering takes place with $\sim 95 \%$ reflection of the electrons at the grain boundaries. Furthermore, the analysis of the transport mobility with respect to grain size in terms of the mean free path shows that, if the grain size of the CdO layers could be increased, electron transport would be significantly improved, leading to better conduction in transparent electrode applications.

\section{ACKNOWLEDGMENTS}

The Engineering and Physical Sciences Research Council, U.K., and the Spanish Government are acknowledged for 
financial support under Grant No. EP/G004447/1 and Grant No. MAT2007-66129, respectively.

${ }^{1}$ F. P. Koffyberg, J. Sol. Stat. Chem. 2, 176 (1970).

${ }^{2}$ D. T. Speaks, M. A. Mayer, K. M. Yu, S. S. Mao, E. E. Haller, and W. Walukiewicz, J. Appl. Phys. 107, 113706 (2010).

${ }^{3}$ M. Yan, M. Lane, C. R. Kannewurf, and R. P. H. Chang, Appl. Phys. Lett. 78, 2342 (2001).

${ }^{4}$ S. Ghosh, A. Sarkar, S. Chaudhuri, and A. Pal, Thin Solid Films 205, 64 (1991).

${ }^{5}$ M. W. J. Prins, K.-O. Grosse-Holz, J. F. M. Cillessen, and L. F. Feiner, J. Appl. Phys. 83, 888 (1998).

${ }^{6}$ D. H. Zhang and H. L. Ma, Appl. Phys. A 62, 487 (1996).

${ }^{7}$ J. Bruneaux, H. Cachet, M. Froment, and A. Messad, Thin Solid Films 197, 129 (1991).

${ }^{8}$ J. Y. W. Seto, J. Appl. Phys. 46, 5247 (1975).

${ }^{9}$ P. D. C. King, T. D. Veal, P. H. Jefferson, J. Zúñiga-Pérez, V. MuñozSanjosé, and C. F. McConville, Phys. Rev. B 79, 035203 (2009).

${ }^{10}$ K. Fuchs, Math. Proc. Cambridge Philos. Soc. 34, 100 (1938).

${ }^{11}$ E. H. Sondheimer, Adv. Phys. 50, 499 (2001).

${ }^{12}$ A. F. Mayadas and M. Shatzkes, Phys. Rev. B 1, 1382 (1970).

${ }^{13}$ D. K. Bowen and B. K. Tanner, High Resolution X-ray Diffractometry and Topography (Taylor and Francis, London, 1998).
${ }^{14}$ A. Piqué, R. C. Y. Auyeung, S. B. Qadri, H. Kim, B. L. Justus, and A. L. Huston, Thin Solid Films 377-378, 803 (2000).

${ }^{15}$ J. Zúñiga-Pérez, C. Munuera, C. Ocal, and V. Muñoz-Sanjosé, J. Cryst. Growth 271, 223 (2004).

${ }^{16}$ C. C. Katsidis and D. I. Siapkas, Appl. Opt. 41, 3978 (2002).

${ }^{17}$ M. Schubert, T. E. Tiwald, and C. M. Herzinger, Phys. Rev. B 61, 8187 (2000).

${ }^{18} \mathrm{H}$. Finkenrath, Physics of II-VI and I-VII Compounds, Semi-Magnetic Semiconductors, Landolt-Börnstein: Numerical Data and Functional Relationships in Science and Technology. Group III: Crystal and Solid State Physics, edited by O. Madelung, M. Schulz, and H. Weiss, Vol. 17B (Springer, Berlin, 1982).

${ }^{19}$ S. G. Choi, J. Zúñiga-Pérez, V. Muñoz-Sanjosé, A. G. Norman, C. L. Perkins, and D. H. Levi, J. Vac. Sci. Technol. B 28, 1120 (2010).

${ }^{20} \mathrm{G}$. Yu, N. L. Rowell, and D. J. Lockwood, J. Vac. Sci. Technol. A 22 , 1110 (2004).

${ }^{21}$ E. O. Kane, J. Phys. Chem. Solids 1, 249 (1957).

${ }^{22}$ P. H. Jefferson, S. A. Hatfield, T. D. Veal, P. D. C. King, C. F. McConville, J. Zúñiga-Pérez, and V. Muñoz-Sanjosé, Appl. Phys. Lett. 92, 022101 (2008).

${ }^{23}$ D. C. Look, H. Lu, W. J. Schaff, J. Jasinski, and Z. Liliental-Weber, Appl. Phys. Lett. 80, 258 (2002).

${ }^{24}$ L. Hsu, R. E. Jones, S. X. Li, K. M. Yu, and W. Walukiewicz, J. Appl. Phys. 102, 073705 (2007). 